\title{
Names of new taxa published and types deposited - A second case study
}

Bandyopadhyay S. ${ }^{1}$, Gopal Krishna ${ }^{1 *}$ and P. Venu ${ }^{2}$

${ }^{1}$ Central National Herbarium, Botanical Survey of India, P.O. Botanic Garden, Howrah - 711 103, West Bengal, India.

${ }^{2}$ Environment Protection Training and Research Institute, 91/4, Gachibowli, Hyderabad - 500 032, Telangana, India.

Received: January 24, 2017; Accepted: February 21, 2017

\begin{abstract}
An attempt has been made to see whether the types of the names of the new taxa published in a botanical journal, Nordic Journal of Botany, for a period of 15 years (1990-2004; volumes 10-24) from India are duly deposited in the cited herbaria of Botanical Survey of India. Earlier a similar exercise was done for the new taxa published in an Indian journal, Rheedea.
\end{abstract}

Key words: Botanical Survey of India; Herbaria; Type deposition; Verification.

\section{Introduction}

In furtherance to our earlier verification exercise on types deposited in designated BSI herbaria for the names published in Rheedea (Bandyopadhyay et al., 2016), the authors repeated the process of verification of the availability of the types of the names of the new taxa published from India in "Nordic Journal of Botany" for the period between 1990 and 2004 (covering volumes 10-24). The way of type verification is the same as followed earier (Bandyopadhyay et al., 2016). However, specimens cited as "additional specimens examined" which are paratypes have not been taken into account if the cited types could be located in the stated herbaria. A total of 50 cases, includng 42 taxa of Angiosperms, 2 species of Lichens, 3 species Fungi and 3 Pteridophytic species were considered for the verification.

The findings are presented below.

\section{Types deposited}

1. Arisaema saddlepeakense P.S.N.Rao \& S.K.Srivast., Nordic J. Bot. 11(5): 575. 1991. (Araceae)

Type: Andaman \& Nicobar Islands, North Andaman, Saddle Peak, 550 m, 2 June 1988, Rao PBL 13170 (holotype, CAL; isotype, PBL).

Note: Holotype found at CAL. Isotype found at PBL.

2. Baubinia ornata Kurz subsp. mizoramensis Bandyop., B.D.Sharma \& Thoth., Nordic J. Bot. 12(2): 223. 1992. (Leguminosae: Caesalpinioideae)

Type: Mizoram, Mizo hills, R. Dutta 33793 (holotype, CAL).

Note: Holotype found at CAL.

3. Beilschmiedia tirunelvelica Manickam, Murugan, Jothi \& Sundaresan, Nordic J. Bot. 24(4): 407. 2007. (Lauraceae)
Type: Tamil Nadu: Tirunelveli district, Agasthiyamalai Biosphere Reserve, Agasthiar Hills, Poongulam, c. 1400m, 24 May 1999, V.S. Manickam 19103 (holotype, $\mathrm{MH}$; isotype, $\mathrm{XCH}$ ); Kanyakumari district, Mahendragiri Hills, on the way to Kailasaparvatam, c. 700 m, 26 March 1999, V.S. Manickam 18652 (paratype, XCH). Note: Holotype found at MH.

4. Clausena austroindica B.C.Stone \& K.Narayanan, Nordic J. Bot. 14(5): 491. 1994. (Rutaceae)

Type: Kerala, Palghat district, from Siruvani Estate, E. Vajravelu 49378 (holotype, CAL; isotypes, CAL, MH).

Note: As per the protologue the holotype should have been deposited at CAL, but it was found housed at MH. Isotypes found at $\mathrm{CAL}$ and $\mathrm{MH}$.

5. Dendrobium gunnarii P.S.N. Rao, Nordic J. Bot. 12(2): 227. 1992. (Orchidaceae)

Type: Andaman \& Nicobar Islands, North Andaman Island, 17.12.1990, P.S.N. Rao 15791 (holotype, CAL; isotype, PBL).

Note: Holotype and isotype found at CAL and PBL, respectively.

6. Eriocaulon raipurense K. K. Khanna, V.Mudgal \& An.Kumar, Nordic J. Bot. 20(4): 413. 2001 (2000 publ. 2001). (Eriocaulaceae)

Type: Madhya Pradesh: Raipur district, Kharara, Choora, 7 October 1976, D.M. Verma 25102 (holotype, CAL); Ambikapur district, Wadroof nagar - Makha Pokhar forest, 22 November 1977, G. Sen Gupta 15693 A (paratype, BSA).

Note: Holotype and paratype found at CAL and BSA, respectively.

\footnotetext{
*Corresponding Author:

Dr. Gopal Krishna,

Central National Herbarium,

Botanical Survey of India, P.O. Botanic Garden,

Howrah - 711 103, West Bengal, India.

E-mail: gopal bsi@yahoo.co.in
}

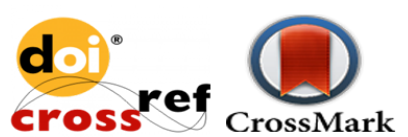


7. Eugenia shettyana Murugan \& Gopalan, Nordic J. Bot. 23(5): 625. 2005. (Myrtaceae)

Type: Kerala: Idukki district, Vaguvarai, Munnar, 2000 $\mathrm{m}, 17.12 .2003$, C. Murugan 117638 (holotype, CAL; isotypes, $\mathrm{MH}, \mathrm{K}$ ); Idukki (Kottayam) district, Umayamalai-Devicolam, 2150 m, 23 November 1965 , B.V. Shetty 26637 (paratypes, MH, K); 2000 m, 18 April 1966, B.V. Shetty 27317; 2150 m, 3 February 1970, B.V. Shetty 33410 (paratypes, MH, K).

Note: Holotype found at CAL. Isotype and paratype found at $\mathrm{MH}$.

8. Garcinia dhanikhariensis S.K.Srivast., Nordic J. Bot. 14(1): 51. 1994. (Clusiaceae)

Type: Andaman \& Nicobar Islands, South Andaman, Nayashahr forest, Dhanikhari Experimental Garden, \pm 30 m, 18 January 1992, fl., S.K. Srivatava 21068 (holotype, CAL; isotype, PBL).

Note: Holotype and isotype found at CAL and PBL, respectively.

9. Gomphia barberi Manickam \& Murugan, Nordic J. Bot. 24(4): 411. 2007. (Ochnaceae)

Type: Tamil Nadu, Tirunelveli district, Kalivagalpil, 1 June 1901, C.A. Barber 3044 (holotype, CAL; isotype, $\mathrm{MH})$.

Note: Holotype and isotype found at CAL and $\mathrm{MH}$, respectively.

10. Hydnocarpus sharmae P.S.N.Rao \& Sreek., Nordic J. Bot. 12(2): 225. 1992. (Flacourtiaceae)

Type: Andaman \& Nicobar Islands, North Andaman, Smith Island, 13 July 1990, P.S.N. Rao 20661 (holotype, CAL; isotype, PBL).

Note: Holotype and isotype found at CAL and PBL, respectively.

11. Parmelina chozoubae K.P. Singh \& G.P. Sinha, Nordic J. Bot. 13(4): 463. 1993.

Type: Nagaland, Phek district, Chakhabama-Zunheboto road, near Chozouba, c. 1575 m, 7 September 1986, K.P. Singh \& G.P. Sinha 2260 (holotype, ASSAM; isotype, US); Nagaland, Wokha district, Mt. Tiyei forest, c. 1450 m, G.P. Sinha 709 (paratype, ASSAM).

Note: Holotype and paratype found at ASSAM.

12. Parmelina nagalandica K.P. Singh \& G.P. Sinha, Nordic J. Bot. 13(4): 464. 1993.

Type: Nagaland: Mokokchung district, near Fazl Ali College, c. 1350 m, 11 April 1985, G.P. Sinha 838 (holotype, ASSAM; isotype, US); Mokokchung-Mariani road, at $3 \mathrm{~km}$ point from Mokokchung town, c. $1300 \mathrm{~m}$, G.P. Sinha 860 (paratype, ASSAM).

Note: Holotype and paratype found at ASSAM.

13. Polyalthia meghalayensis V.Prakash \& Mehrotra, Nordic J. Bot. 10(1): 45. 1990. (Annonaceae)

Type: Meghalaya, Garo Hills district, Tura peak, Boldoringri forest, 21 April 1987, Ved Prakash 17130 A (holotype, CDRI; isotypes, Ved Prakash 17130 B, K; Ved Prakash 17130 C, CAL).

Note: Isotype found at CAL.
14. Sporobolus hajrae P.Umam. \& P. Daniel, Nordic J. Bot. 18(5): 577. 1998. (Poaceae)

Type: Tamil Nadu, Chidambaranar distrct, Veppalodai seacoast, c. $25 \mathrm{~km}$ from Tuticorin, 30 July 1995, P. Daniel \& P. Umamahaswari 103594 (holotype, CAL; isotype, $\mathrm{MH})$.

Note: Holotype and isotype found at CAL and $\mathrm{MH}$, respectively.

\section{Types partly deposited}

1. Antistrophe glabra Pandur. \& V. J. Nair, Nordic J. Bot. 17(4): 367. 1997. (Myrsinaceae)

Type: Kerala, Idukki district, Kanjiar, c. 1000 m, 10.10.1983, A.G. Padurangan 79291 (holotype, CAL; isotype MH); Kanjiar, c. $1000 \mathrm{~m}, 30.12 .1983$, A.G. Padurangan 80090 (paratype, MH).

Note: Holotype not found at CAL. Isotype and paratype found at $\mathrm{MH}$.

2. Epipogium indicum H.J.Chowdhery, G.D.Pal \& G.S.Giri, Nordic J. Bot. 13(4): 419. 1993. (Orchidaceae)

Type: Arunachal Pradesh, Lower Subansiri district, Sankei View, 500-600 m, 14.4.1992, H.J. Chowdhery 1746 (holotype, CAL; isotype, ARUN).

Note: Holotype not found at CAL. Isotype found at ARUN.

3. Eriocaulon balakrishnanii Punekar, Lakshmin. \& Vasudeva Rao, Nordic J. Bot. 22(5): 609. 2003 (2002, publ. 2003). (Eriocaulaceae)

Type: Karnataka, Uttara Kannada district, Anshi N.P., Tiger tank, c. $2 \mathrm{~km}$ from Anshi village, $600 \mathrm{~m}$ above msl, 26 January 2002, Sachin A. Punekar 183420 A (holotype, CAL; isotypes, Sachin A. Punekar 183420 B, MH; Sachin A. Punekar 183420 C-E, BSI); ibid., 31 March 2002, Sachin A. Punekar 183489 A-C (paratypes, MH).

Note: Holotype and isotypes found at CAL and BSI, respectively. Isotype and paratypes not found at $\mathrm{MH}$.

4. Eriocaulon peninsulare Punekar \& Lakshmin., Nordic J. Bot. 23(2): 173. 2004. (Eriocaulaceae)

Type: Karnataka, Uttara Kannada district, Anshi National Park, Tiger tank, c. $2 \mathrm{~km}$ from Anshi village, $600 \mathrm{~m}$ above msl, 26 January 2002, Sachin A. Punekar 183423 (holotype, CAL); Sachin A. Punekar 183420 B (isotypes, $\mathrm{MH}, \mathrm{BSI}$ ).

Note: Holotype and isotypes found at CAL and BSI, respectively. Another isotype not found at $\mathrm{MH}$.

5. Pholidota pygmaea H.J.Chowdhery \& G.D.Pal, Nordic J. Bot. 15(4): 411.1996 (1995 publ. 1996). (Orchidaceae)

Type: Arunachal Pradesh, Lower Subansiri district, Itanagar, $600 \mathrm{~m}$, 30.7.1993, H.J. Chowdhery 1760 (holotype, CAL; isotype, ARUN).

Note: Holotype not found at CAL. Isotype found at ARUN.

6. Pogostemon hedgei V.S.Kumar \& B.D.Sharma, Nordic J. Bot. 15(2): 163. 1995. (Lamiaceae)

Type: Tamil Nadu, Kanniyakumari district, Muthukuzhivayal way, 1 February 1978, A.N. Henry 
53351 A (holotype, MH; isotypes, A.N. Henry 53351 B, CAL, A.N. Henry 53351 C, MH).

Note: Holotype and isotype found at MH. Isotype not found at CAL.

7. Pogostemon speciosus Benth. var. filiformis V.S.Kumar \& B.D.Sharma, Nordic J. Bot. 15(2): 166. 1995. (Lamiaceae)

Type: Tamil Nadu, Nilagiri district, Thai shola, 31 January 1913, C.E.C. Fischer 3540 (holotype, CAL); Nilagiri district, Carnington, 5 January 1971, B.V. Shetty 37715 (paratype MH).

Note: Holotype not found at CAL. Paratypes found at $\mathrm{MH}$.

8. Psychotria henryana Murugan \& Gopalan, Nordic J. Bot. 24(4): 415. 2007. (Rubiaceae)

Type: Tamil Nadu, Kanyakumari district, Muthukuzhi, A.N. Henry 52454 (holotype, CAL; isotype, MH).

Note: Holotype not found at CAL (as per record the holotype has been received at CAL but could not be found in type section). Isotype found at $\mathrm{MH}$.

9. Ranunculus uttaranchalensis Pusalkar \& D.K.Singh, Nordic J. Bot. 24(4): 403. 2007. (Ranunculaceae)

Type: Western Himalaya, Uttaranchal, Uttarkahi, Gangotri National Park, upper Nandanvan, near Bhagirathi II base camp, 15 July 2003, Pusalkar 102334 (holotype, BSD; isotype, CAL).

Note: Holotype found at BSD. Isotype not found at CAL.

10. Trichodesma mudgalii An.Kumar \& K.K.Khanna, Nordic J. Bot. 22(2): 223. 2003 (2002 publ. 2003). (Boraginaceae)

Type: Madhya Pradesh, Betul distict, Atner, 16.4.1997, Anand Kumar 50039 A (holotype, CAL); Anand Kumar 50039 B (isotype, CAL); Anand Kumar 50039 C (isotype, BSA).

Note: Holotype found at CAL. Isotype found only at BSA, not at CAL. In the protologue Anand Kumar 50039 $\mathrm{B}$ and $\mathrm{C}$ have been cited as 'paratypes'.

\section{Types not deposited/could not be traced}

1. Acrotrema agastyamalayanum E.S.S.Kumar, Dan \& G.M.Nair, Nordic J. Bot. 24(5): 543. 2007. (Dilleniaceae) Type: Kerala, Thiruvananthapuram district, Braemore, \pm 500 m, 12.11.2002, Santhosh Kumar 48414 (holotype, TBGT; isotypes, CAL, MH).

Note: Isotypes not found at CAL and MH.

2. Amomum jainii S.Tripathi \& V.Prakash, Nordic J. Bot. 19(5): 609. 1999. (Zingiberaceae)

Type: Meghalaya, Garo Hills, Bagmara forest, 8 May 1997, Sunil Tripathi 20827 A (holotype, CDRI; Sunil

Tripathi 20827 B, isotype, CAL).

Note: Isotype not found at CAL.

3. Andrographis chendurunii E.S.S.Kumar, A.E.S.Khan \& S.G.Gopal, Nordic J. Bot. 22(6): 683. 2004. (Acanthaceae)
Type: Kerala, Kollam district, Pandimotta, 950 m, 11 January 1994, Santhosh Kumar 19173 (holotype, TBGT; isotypes, CAL, $\mathrm{MH}, \mathrm{K}$ ).

Note: Isotypes not found at CAL and $\mathrm{MH}$.

4. Ceropegia schumanniana Swarupan. \& Mangaly, Nordic J. Bot. 12(6): 699. 1992. (Asclepiadaceae)

Type: Tamil Nadu, Coimbatore district, Kharian Shola, near Top Slip, sterile, 1.5.1976, fl., 29.12.1976 (transplant), Swarupanandan 13911 (holotype, MH).

Note: Holotype not found at MH.

5. Cheirostylis gunnarii A.N.Rao, Nordic J. Bot. 18(1): 23. 1998. (Orchidaceae)

Type: Arunachal Pradesh, Lohit district, Kamlang

Reserve forest, $300 \mathrm{~m}$, on moss covered tree trunks, 16 March 1996, A.N. Rao 28003 (holotype, Orchid

Herbarium, Tipi); [no details provided] A.N. Rao 28004 (paratype, CAL); [no details provided] A.N. Rao 28005 (paratype, ASSAM).

Note: Paratypes not found at CAL and ASSAM.

6.Coelogyne arunachalensis H.J.Chowdhery \& G.D.Pal, Nordic J. Bot. 17(4): 369. 1997. (Orchidaceae)

Type: Arunachal Pradesh, Lower Subansiri district, near Doimukh, on way to Sagali, 500-800 m, 2.11.1985, G.D. Pal 1790 (holotype, CAL).

Note: Holotype not found at CAL.

7. Crepidomanes agasthianum Madhus. \& C.A.Hameed, Nordic J. Bot. 18(2): 169. 1998. (Hymenophyllaceae)

Type: Kerala, Thiruvananthapuram district, Agasthiamalai, $1000 \mathrm{~m}, 13$ May 1993, P.V.Madhusoodanan, Santhosh Nampy \& A.K. Pradeep CU 51906 (holotype, CALI; isotypes, K, CAL).

Note: Isotype not found at CAL.

8.Crepidomanes malabaricum C.A.Hameed \& Madhus., Nordic J. Bot. 19(3): 277. 1999. (Hymenophyllaceae)

Type: Kerala, Trichur district, Athirapally forest, $700 \mathrm{~m}$, 21 December 1997, C. Abdul Hameed CU 34858 (holotype, CALI; isotypes, $\mathrm{K}, \mathrm{MH}$ ).

Note: Isotype not found at $\mathrm{MH}$.

9. Curcuma prakasha S.Tripathi, Nordic J. Bot. 21(5): 549. 2002 (2001 publ. 2002). (Zingiberaceae)

Type: Meghalaya, Garo Hills, Bagmara district, Sidgiri, 6 May 1997, Sunil Tripathi 20825 (holotype, CDRI; isotype, CAL).

Note: Isotype not found at CAL.

10. Fuirena simpsonii Ravi, N.Mohanan \& Shaju, Nordic J. Bot. 22(3): 329. 2003 (2002 publ. 2003). (Cyperaceae)

Type: Kerala, Kannur district, Pappinisseri, 12 December 1997, Ravi 37077 (holotype, TBGT; isotypes, CAL, TBGT).

Note: Isotype not found at CAL.

11. Hornstedtia arunachalensis S.Tripathi \& V. Prakash, Nordic J. Bot. 19(3): 329. 1999. (Zingiberaceae) 
Type: Arunachal Pradesh, Papampara district, Umpham, 1 May 1997, Sunil Tripathi 20849 (holotype, CDRI; isotype, CAL).

Note: Isotype not found at CAL.

12. Ixora sivarajiana Pradeep, Nordic J. Bot. 17(3): 315. 1997. (Rubiaceae)

Type: Kerala, Kozhikode district, Vellarimala, 2240 m, 10 April 1995, Pradeep 5365 (holotype K; isotypes MH, CALI).

Note: Isotype not found at MH.

13. Litsea beei N.Mohanan \& E.S.S.Kumar, Nordic J. Bot. 23(5): 611. 2005. (Lauraceae)

Type: Kerala: Thiruvananthapuram district, Agasthiamala Hills, Attayar, c. 700 m, 27th June 1994, Mohanan TBG \& RI 12439 (holotype, TBGT; isotypes, CAL, MH); Thiruvananthapuram district, Agasthyamala Hills, Bonaccord, 650 m, 27th June 1994, Raj Kumar TBG \& RI 11760 (paratype, TBGT); ibid., Attayar, 700 m, 11th January 1996, Santhosh Kumar TBG \& RI 26836 (paratype, TBGT).

Note: Isotypes not found at CAL and MH.

14. Lygodium andamanicum R.D.Dixit, J.B.Bhandari \& R.Mukhop., Nordic J. Bot. 21(4): 427. 2002 (2001 publ. 2002). (Lygodiaceae)

Type: Andaman \& Nicobar Islands, North Andamans, Saddle Peak, c. 650 m, December 1992, Dixit 53396 (holotype, CAL; [no details provided] (paratype BU).

Note: Holotype not found at CAL.

15. Memecylon bremeri M.B.Viswan., Nordic J. Bot. 21(3): 256. 2001. (Melastomataceae)

Type: Tamil Nadu, Salem district, Kolli hills, $11^{\circ} 8^{\prime} \mathrm{N}$ and $78^{\circ} 20^{\prime}$ E, Karavallicombai R.F., \pm 1200 m, 2 April 1994, M.B. Viswanathan 3 (holotype, MH; isotypes, CAL, herbarium at Sri Paramakalyani Centre for Environmental Sciences, Alwarkurichi, Tirunelveli).

Note: Holotype not found at MH. Isotype not found at CAL. Ms. Moumita Das Das who is revising the Indian Memecylons has kindly informed that she could not locate the type in the herbarium at Sri Paramakalyani Centre for Environmental Sciences, Alwarkurichi, Tirunelveli.

16. Memecylon kollimalayanum M.B.Viswan., Nordic J. Bot. 21(3): 253. 2001. (Melastomataceae)

Type: Tamil Nadu, Salem district, Kolli hills, 11 ${ }^{\circ} 8^{\prime} \mathrm{N}$ and $78^{\circ} 20^{\prime}$ E, Karavallicombai R.F., \pm 1200 m, 2 April 1994, M.B. Viswanathan 2 (holotype, MH; isotypes, CAL, herbarium at Sri Paramakalyani Centre for Environmental Sciences, Alwarkurichi, Tirunelveli); ibid., 15 June 1995, M.B. Viswanathan 17240 (paratypes, herbarium at Sri Paramakalyani Centre for Environmental Sciences, Alwarkurichi, Tirunelveli).

Note: Holotype and isotype not found at MH and CAL, respectively. Ms. Moumita Das Das informed that she could not locate the types in the herbarium at Sri Paramakalyani Centre for Environmental Sciences, Alwarkurichi, Tirunelveli.
17. Memecylon mundanthuraianum M.B.Viswan. \& Manik., Nordic J. Bot. 21(3): 259. 2001. (Melastomataceae)

Type: Tamil Nadu, Kalakkad-Mundanthurai Tiger reserve, $8^{\circ} 20^{\prime}-8^{\circ} \quad 53^{\prime} \mathrm{N}$ and $77^{\circ} 10^{\prime} \mathrm{N}-77^{\circ} 35^{\prime} \mathrm{E}$, Kodamadi, \pm 450 m, 9 March 2000, M.B. Viswanathan \& U. Manikandan 4282 (holotype, MH; isotypes, CAL, herbarium at Sri Paramakalyani Centre for Environmental Sciences, Alwarkurichi, Tirunelveli); ibid., M.B. Viswanathan \& U. Manikandan 3393 (paratypes, herbarium at Sri Paramakalyani Centre for Environmental Sciences, Alwarkurichi, Tirunelveli).

Note: Holotype and isotype not found at $\mathrm{MH}$ and CAL, respectively. Ms. Moumita Das Das informed that she could not locate the types in the herbarium at Sri Paramakalyani Centre for Environmental Sciences, Alwarkurichi, Tirunelveli.

18. Memecylon sivadasanii N.Mohanan, Ravi, Kiran Raj \& Shaju, Nordic J. Bot. 21(5): 493. 2002 (2001 publ. 2002). (Melastomataceae)

Type: Kerala, Thiruvananthapuram district, Agasthyamala Hills, Kurisumala, Bonaccord, c. 900 m, 17th March 1993, Mohanan TBG \& RI 11313 (holotype, TBGT; isotypes, $\mathrm{K}, \mathrm{MH}$ ).

Note: Isotype not found at $\mathrm{MH}$.

19. Neurocalyx bremeri M.B.Viswan., Manik. \& Tangav., Nordic J. Bot. 23(4): 389. 2005. (Rubiaceae)

Type: Tamil Nadu, Kalakkad-Mundanthurai Tiger Reserve, $8^{\circ} 20^{\prime}-8^{\circ} 53^{\prime} \mathrm{N}$ and $77^{\circ} 10^{\prime}-77^{\circ} 35^{\prime} \mathrm{E}$, Inchikuli, 750 m, 3 December 2002, M.B. Viswanathan \& U. Manikandan 9085 (holotype, MH; isotypes, herbarium at Sri Paramakalyani Centre for Environmental Sciences, Alwarkurichi, Tirunelveli); ibid., 4 November 2000, M.B. Viswanathan \& U. Manikandan 6895 (paratypes, herbarium at Sri Paramakalyani Centre for Environmental Sciences, Alwarkurichi, Tirunelveli).

Note: Holotype not found at $\mathrm{MH}$.

20. Nymphoides krishnakesara K.T.Joseph \& Sivar., Nordic J. Bot. 10(3): 281. 1990. (Menyanthaceae)

Type: Kerala, Cannanore district, Madai, October 1988, Joseph 43001 (Holotype, MH; isotypes, Z, CALI).

Note: Holotype not found at $\mathrm{MH}$.

21. Orophea sivarajanii Sasidh., Nordic J. Bot. 19(3): 301. 1999. (Annonaceae)

Type: Kerala, Wyanad district, Periya Reserve Forests, \pm $800 \mathrm{~m}$, 20th December 1994, Sasidharan 10844 (holotype, MH; isotypes, L, KFRI, CALI).

Note: Holotype not found at $\mathrm{MH}$.

22. Polycarpaea majumdariana Venu, Muthuk. \& P.Daniel, Nordic J. Bot. 21(6): 579. 2002 (2001 publ. 2002). (Caryophyllaceae)

Type: Tamil Nadu, Tirunelveli district, Seethaparpanallur, c. $15 \mathrm{~km}$ from Tiruvelveli town on Tensaki road, c. 120 m, fl. \& fr., 28 December 1998, Muthukumar 15 (holotype, CAL; isotypes, $\mathrm{K}, \mathrm{MH}$ ).

Note: Holotype and isotype not found at CAL and $\mathrm{MH}$, respectively. 
23. Selaginella camusiae Antony \& E.S.S.Kumar, Nordic J. Bot. 22(3): 337.2003 (2002 publ. 2003). (Selaginellaceae) Type: Kerala, Thiruvananthapuram district, Agastyamala, 1750 m, 9 January 1996, Raju Antony 25581 (holotype, TBGT; isotypes, CALI, MH).

Note: Isotype not found at MH.

24. Selaginella dixitii Madhus. \& S. Nampy, Nordic J. Bot. 14(5): 527. 1994. (Selaginellaceae)

Type: Kerala, Palakkad district, Nelliampathy, 700 m, 28 December 1992, P.V. Madhusoodanan \& Santhosh Nampy CU 48898 (holotype, CALI; isotypes, K, CAL).

Note: Isotype not found at CAL.

25. Trichomanes vamana C. A. Hameed \& Madhus., Nordic J. Bot. 23(4): 439. 2005. (Hymenophyllaceae)

Type: Kerala, Idukki district, Thommankuthu stream, 27 December 1996, C. Abdul Hameed \& Santhosh Nampy CU 34814 (holotype, CALI; isotypes, $\mathrm{K}, \mathrm{MH}$ ).

Note: Isotype not found at MH.

26. Viscum malurianum Sanjai \& N.P.Balakr., Nordic J. Bot. 21(3): 267. 2001. (Viscaceae)

Type: Karnataka, Malur, $850 \mathrm{~m}$, near the Railway Station on Ficus benghalensis, 20 October 1995, Sanjai 104911 (holotype, CAL; isotype, MH).

Note: Holotype and isotype not found at CAL and MH, respectively.

\section{Conclusion}

The analysis suggests that the types of 14 names exist at the stated herbarium / herbaria; types are partly found for 10 names; no types could be located in the remaining 26 cases. For 15 names holotypes are not found and for 6 names, there are no types at all.

\section{Acknowledgements}

We thank Dr. Paramjit Singh, Director, Botanical Survey of India (BSI), for approving this study as project work. We also thank Dr. S.K. Srivastava, (NRC, Dehradun); Dr. G.V.S. Murthy and Dr. J.V. Sudhakar (SRC, Coimbatore); Dr. B.K. Sinha (ERC, Shillong); Dr. C. Murugan, (ANRC, Port Blair) and Dr. J. Jayanthi (WRC, Pune), for facilitating the information on the type specimens. Dr. W. Arisdason, Central National Herbarium, BSI, helped to improve the manuscript.

\section{Reference}

1. Bandyopadhyay S., Gopal Krishna and P. Venu. Names of new taxa published and types deposited A case study. Annals of Plant Sciences 5.10 (2016): 1451-1457.

\section{Cite this article as:}

Bandyopadhyay S., Gopal Krishna and P. Venu. Names of new taxa published and types deposited - A second case study. Annals of Plant Sciences 6.03 (2017): 1585-1589.

DOI: http://dx.doi.org/10.21746/aps.2017.03.002 